\title{
Pure Quasi- $P$-wave calculation in transversely isotropic media using a hybrid method
}

\author{
Zedong $\mathrm{Wu}^{1}$, Hongwei $\mathrm{Liu}^{2}$ and Tariq Alkhalifah ${ }^{1}$ \\ 1 Seismic wave analysis group, King abdullah university of science and technology, Thuwal, Saudi Arabia \\ ${ }^{2}$ EXPEC Advanced Research Center, Saudi Aramco, Saudi Arabia
}

\section{SUMMARY}

The acoustic approximation for anisotropic media is widely used in current industry imaging and inversion algorithms mainly because P-waves constitute the majority of the energy recorded in seismic exploration. The resulting acoustic formulas tend to be simpler, resulting in more efficient implementations, and depend on fewer medium parameters. However, conventional solutions of the acoustic wave equation with higher-order derivatives suffer from shear wave artifacts. Thus, we derive a new acoustic wave equation for wave propagation in transversely isotropic (TI) media, which is based on a partially separable approximation of the dispersion relation for TI media and free of shear wave artifacts. Even though our resulting equation is not a partial differential equation, it is still a linear equation. Thus, we propose to implement this equation efficiently by combining the finite difference approximation with spectral evaluation of the space-independent parts. The resulting algorithm provides solutions without the constrain of $\epsilon \geq \delta$. Numerical tests demonstrate the effectiveness of the approach.

Key words: VTI, Acoustic. 


\section{INTRODUCTION}

The acoustic approximation is widely used in current industry imaging and inversion algorithms (Gholami et al. 2013; Alkhalifah \& Plessix 2014; Cheng et al. 2014a,b; Operto et al. 2015; da Silva et al. 2016). This approximation was introduced by Alkhalifah (2000) for the purpose of resolving quasi-P wave propagation in transversely isotropic (TI) media. In his approach, the shear-wave velocity along the symmetry axis is set to zero, resulting in a scalar fourth-order differential equation. Zhou et al. (2006) decomposed the fourth-order differential equation into a coupled system of second-order differential equations, and proposed a computationally efficient scheme. After that, several variations (Bakker \& Duveneck 2011; Zhang et al. 2011; Bube et al. 2012) were introduced to improve stability and efficiency. However, most of them admit $S$-wave artifacts in the resulting wavefield. These artifacts are reduced when the source is located in an isotropic layer (Alkhalifah 2000), but become unacceptable when the source is located in the anisotropic region or we have strong scattering acting as secondary sources(Wu \& Alkhalifah 2015; Alkhalifah \& Wu 2016; Wu \& Alkhalifah 2017).

One family of high resolution algorithms for solving the anisotropic acoustic wave equation free of $S$-wave artifacts falls under the so-called spectral approach (Etgen \& Brandsberg-Dahl 2009; Du et al. 2010; Fomel et al. 2013; Alkhalifah 2014; Song \& Alkhalifah 2013; Wu \& Alkhalifah 2014; Sun et al. 2016). They are based on approximately separating the space- and wavenumber-dependency of the dispersion relation. Numerically, they are reasonably accurate and admit no $S$-wave artifacts. However, they usually require a lot of Fourier transforms for each time step in the case of complicated heterogeneous media. This makes these kinds of methods relatively expensive.

Another group of efficient algorithms for computing pure quasi-P waves is the so-called effective isotropic model approximation (Alkhalifah et al. 2013; Ibanez-Jacome et al. 2014; Waheed \& Alkhalifah 2015). These approaches perform the quasi-P wave calculation in two steps. First by solving the Eikonal equation from an anisotropic quasi-P wave velocity model, thereby obtaining the propagation direction at each spatial point; this allows the determination of the phase velocity and the formation of an effective model for quasi-P wave propagation. Then an isotropic wave propagator is used to obtain the solution. The computational cost of the effective isotropic model approach is close to that of solving an isotropic acoustic wave equation, however, it is accurate mainly for the first arrivals as the eikonal equation dictates.

Recently, and along the lines of the effective isotropic model approximation, Xu \& Zhou (2014) proposed a new dynamic effective model equation that decompose the original pseudodifferential operator into two numerically solvable operators: a Laplacian operator and a scalar 
operator. The combination of these two operators yields an accurate phase for quasi-P wave propagation. This solution is shear-wave free and numerically stable even for complicated anisotropic models. Since only one equation is required to obtain a numerical solution, the new proposed scheme is more efficient than conventional schemes that solve a system of secondorder differential equations. In order to compensate for amplitude errors, Xu et al. (2015) proposed decomposing the original operator into elliptic anisotropic and anelliptic anisotropic components. In order to reduce the cost, they suggest doing so in the time domain. Le et al. (2015) applied their method to full waveform inversion. To speed up the computation, Zhang et al. (2017) proposed to use the low rank approximation for the isotropic components of the resulting equation in Xu \& Zhou (2014).

In $\mathrm{Wu} \&$ Alkhalifah (2018), the authors proposed a partially separable approximation to the anelliptic anisotropic component for the acoustic wave equation in VTI media described in the frequency domain. As such, they used the inverse matrix of the discrete approximation of the acoustic wave equation in elliptic anisotropic media as a preconditioner of the approximate matrix of the acoustic wave equation for VTI media.

Most of the above mentioned algorithms are essentially designed for the case of $\epsilon-\delta>0$. This assumption has been widely adopted in production from model building to pseudoacoustic seismic imaging and modeling in Vertical Transversely Isotropic (VTI) and Tilted Transversely Isotropic (TTI) media. This is because lab measurements show that $\epsilon$ is usually greater than $\delta$ for shales. In contrast to shales, $\epsilon$ is usually smaller than $\delta$ in carbonate rocks (Wang 2002; Baden et al. 2017) and in this case, the traditional pseudo-acoustic reverse time migration (RTM) fails. Elastic RTM is an option, but it is much more expensive than acoustic RTM and there is still no good way to build elastic models in production.

In this paper, we derive an efficient implementation of wave propagation in acoustic transversely isotropic (TI) media free of $S$-wave artifacts. We first derive a new wave equation in acoustic transversely isotropic (TI) media based on a partially separable approximation of the dispersion relation. This separable approximation is reasonably accurate for the case where $\epsilon-\delta$ is negative or positive. The resulting formula is linear, allowing us to utilize the Fourier domain to evaluate the space stationary terms. After that, we propose an efficient numerical implementation that combines finite difference discretization and Fast Fourier transform. At last, several numerical examples are shown to illustrate the accuracy of the proposed method for simple and complicated models. 


\section{A PARTIALLY SEPARABLE APPROXIMATION TO VTI MEDIA}

The dispersion relation for acoustic VTI media in the frequency-wavenumber domain (Alkhalifah 2000; Fomel et al. 2013) can be represented as follows:

$$
\omega^{2}=\frac{v^{2}}{2}\left[(1+2 \epsilon) k_{x}^{2}+k_{z}^{2}+\sqrt{\left((1+2 \epsilon) k_{x}^{2}+k_{z}^{2}\right)^{2}-8(\epsilon-\delta) k_{x}^{2} k_{z}^{2}}\right]
$$

in which, $\omega$ is the angular frequency, $\epsilon$ and $\delta$ are the familiar Thomsen parameters (Thomsen 1986) and $v$ is the velocity along symmetry axis. The spatial wavenumber vector $\vec{k}$ is, as usual, defined as $\vec{k}=\left\{k_{x}, k_{z}\right\}$ in $2 \mathrm{D}$ media. We can reformulate the dispersion relation as:

$$
\omega^{2}=(F(\vec{k}, \vec{p})+1)\left[v^{2} k_{z}^{2}+v^{2}(1+2 \epsilon) k_{x}^{2}\right]
$$

with $F$ given by

$$
F(\vec{k}, \vec{p})=\frac{1}{2}\left(\sqrt{1-\frac{8(\epsilon-\delta) k_{x}^{2} k_{z}^{2}}{\left((1+2 \epsilon) k_{x}^{2}+k_{z}^{2}\right)^{2}}}-1\right) .
$$

Let us denote

$$
m=\frac{8(\epsilon-\delta) k_{x}^{2} k_{z}^{2}}{\left(k_{x}^{2}+k_{z}^{2}\right)^{2}}, n=\frac{2 \epsilon k_{x}^{2}}{\left(k_{x}^{2}+k_{z}^{2}\right)}
$$

then

$$
F=\frac{1}{2}\left(\sqrt{1-\frac{m}{(1+n)^{2}}}-1\right)
$$

The key problem in the above formulation is that $F$ is a function of both space and wavenumber. In this case, we can not simply utilize fast Fourier transform for the implementation of the operator $F$. Thus, we need to approximate $\mathrm{F}$ in some kind of a separable form:

$$
F_{a 0}=\sum_{i=1}^{p} f_{i}(m) g_{i}(n) .
$$

To do that, we can seek the following approximation of $F$

$$
F_{a 1}=b_{1} m^{b_{2}}+b_{3} m^{b_{4}} n^{b_{5}} .
$$

It provides a good approximation when $\epsilon-\delta$ is either non-negative or non-positive. However, in the case of a model which has $\epsilon-\delta$ both positive and negative, it does not provide very good accuracy. To remedy this issue, we propose a natural extension of (7) given by:

$$
F_{a}=H(m)\left(b_{1}|m|^{b_{2}}+b_{3}|m|^{b_{4}} n^{b_{5}}\right)+(1-H(m))\left(b_{6}|m|^{b_{7}}+b_{8}|m|^{b_{9}} n^{b_{10}}\right) .
$$

In the above formulation, $H(m)$ is a Heaviside function, which is defined as:

$$
H(m)=1, \forall m \geq 0 ; H(m)=0, \forall m<0 .
$$

Note that the above functions are all continuous even though the Heaviside function itself is not continuous. If we assume that $b_{2}>0, b_{4}>0, b_{7}>0$ and $b_{9}>0 .\left\{b_{i}\right\}_{i=1}^{10}$ are the coefficients 
to be decided. Actually, we can choose $b_{1}=b_{6}=-\frac{1}{4}, b_{2}=b_{7}=0, b_{3}=b_{8}=-0.5, b_{4}=b_{9}=$ $0, b_{5}=b_{10}=1$, then

$$
F_{a}=-\frac{m}{4}+\frac{m n}{2}
$$

which reduces to a Taylor series expansion of $F$ over the two variables $m$ and $n$. It is a good approximation of $F$ when $m$ and $n$ are relatively small. To make the approximation more accurate for a larger range of $m$ and $n$, we need to search for the best $\left\{b_{i}\right\}_{i=1}^{10}$. Considering that $(m, n) \in \Omega$, the coefficients can be decided through solving the following optimization problem:

$$
\min _{b_{1}, b_{2}, b_{3}, b_{4}, b_{5}, b_{6}, b_{7}, b_{8}, b_{9}, b_{10}} \max _{(m, n) \in \Omega}\left|F-F_{a}\right| .
$$

Here we assume that $b_{2}>0, b_{4}>0, b_{7}>0$ and $b_{9}>0$. In addition, $F_{a}=0$ when $m=0$. In this special case, the original dispersion relation reduces to the elliptic anisotropic wave equation and our approximate dispersion relation is the same as the original dispersion $F\left(F_{a}=F\right)$. It indicates that there is no approximation error in the case of the elliptic anisotropic wave equation. The accuracy of the approximation is demonstrated in Table 1 for different ranges of $m$ and $n$. Compared with the standard Taylor series expansion, the optimal coefficients are much more accurate for the same range of $m$ and $n$, especially for relatively large $m$ and $n$. Comparing the dispersion error of the conventional finite difference method of 8 th order even for the isotropic wave equation, which is about 0.005 , our maximum dispersion error is neglegible for even $|m| \leq 0.6$ and $n \leq 0.6$. For additional comparison, we show the exact $F$ for different $m$ and $n$ in Figure 1a. The error of the Taylor-based (proposed) approximation is shown in Figure 1b. We can see from Figure 1b and 1c that the proposed approximation is much more accurate than the approximation based on the Taylor series expansion. Figures $1 \mathrm{a}, 1 \mathrm{~b}$, and $1 \mathrm{c}$ are plotted using the same scale. To show the error distribution, we increase the error distribution of the proposed method by a factor of 10 and show it in Figure 1d. At last, the choice of $\Omega$ can be decided by the range of $\epsilon$ and $\delta$. If $|\epsilon-\delta| \leq \alpha$ for a given $\alpha$, then $|m|=\left|\frac{8(\epsilon-\delta) k_{x}^{2} k_{z}^{2}}{\left(k_{x}^{2}+k_{z}^{2}\right)^{2}}\right| \leq 2 \alpha$. If $\epsilon \leq \beta$, then $n=\frac{2 \epsilon k_{x}^{2}}{\left(k_{x}^{2}+k_{z}^{2}\right)} \leq 2 \beta$.

\section{APPROXIMATE ACOUSTIC WAVE EQUATION IN VTI MEDIA}

Since we have obtained a separable approximation, we will now formulate the relative equation in the time-space domain. According to the above derivation, the approximate dispersion relation is as follows:

$$
L_{1}(\vec{k}, \vec{p}, \omega)=\omega^{2}-\left(v^{2} k_{z}^{2}+v^{2}(1+2 \epsilon) k_{x}^{2}\right)-F_{a}(\vec{k}, \vec{p})\left(v^{2} k_{z}^{2}+v^{2}(1+2 \epsilon) k_{x}^{2}\right) .
$$




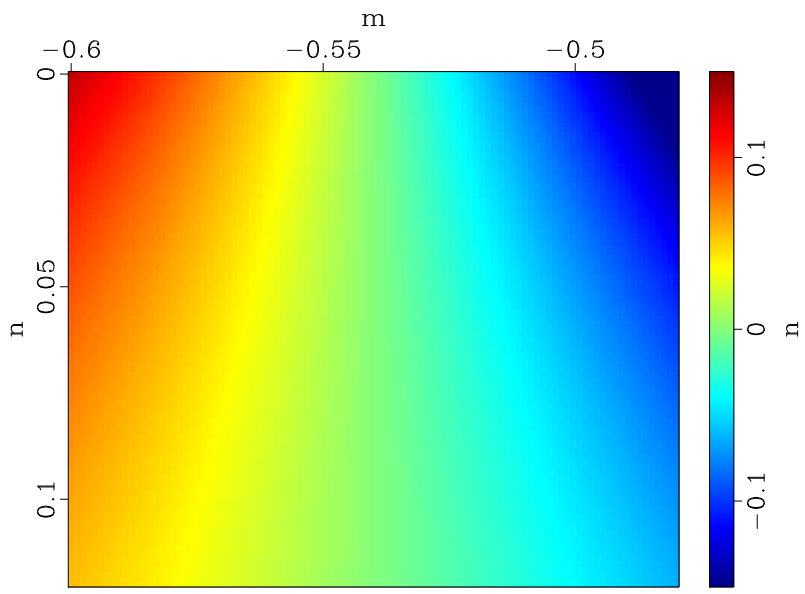

(a)

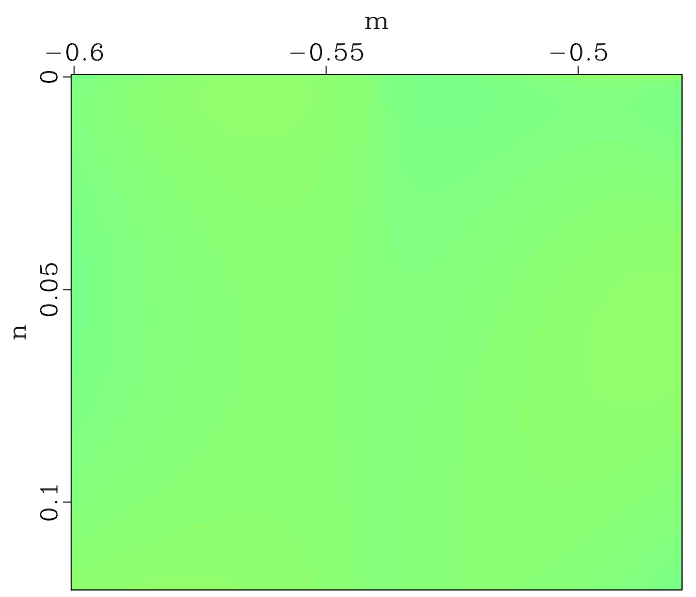

(c)

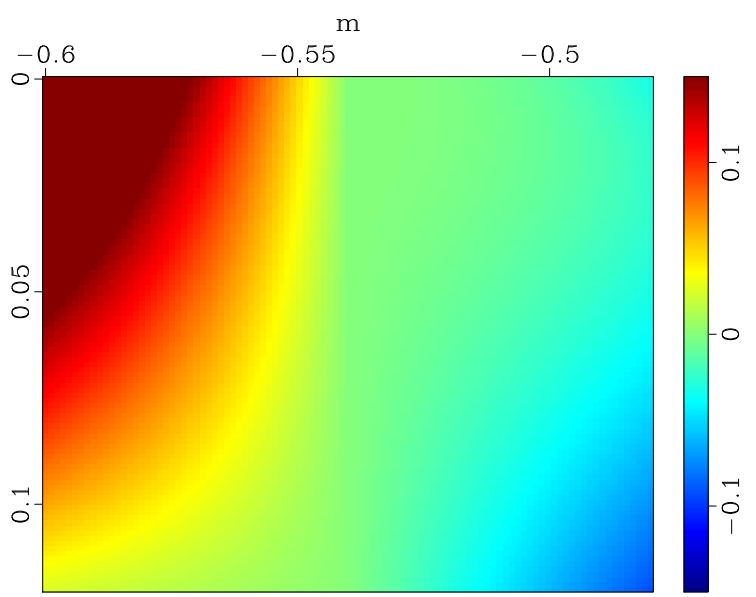

(b)

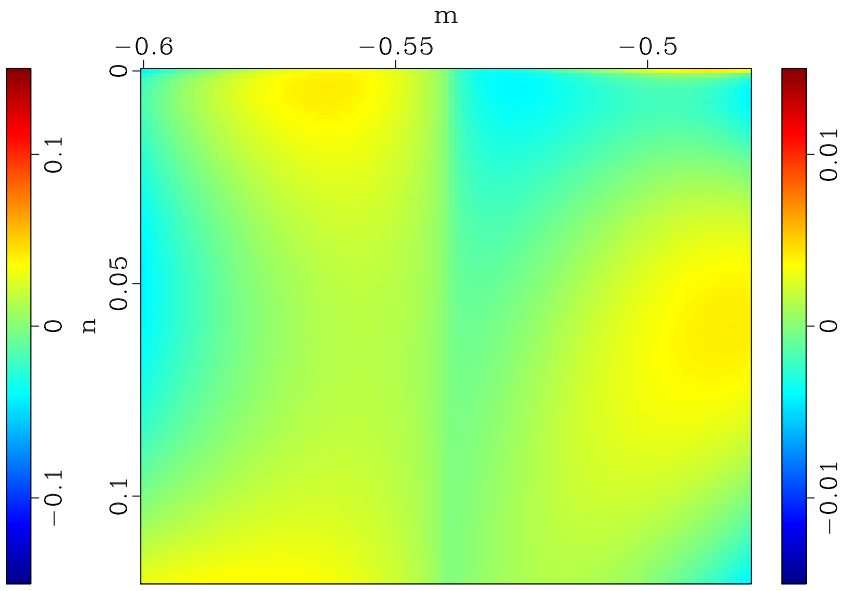

(d)

Figure 1. Synthetic example showing (a) the exact formulation F, (b) the approximation error of the Taylor expansion-based method, (c) the approximation error of the proposed method, and (d) The approximation error of the proposed method with $10 \%$ clip of that in (a), (b) and (c).

We apply an inverse Fourier transform from frequency-wavenumber to time-space, and obtain the following equation:

$$
u_{t t}-\left(v^{2} \frac{\partial^{2} u}{\partial z^{2}}+v^{2}(1+2 \epsilon) \frac{\partial^{2} u}{\partial x^{2}}\right)-F_{a}(\vec{k}, \vec{p})\left(v^{2} \frac{\partial^{2} u}{\partial z^{2}}+v^{2}(1+2 \epsilon) \frac{\partial^{2} u}{\partial x^{2}}\right)=f .
$$

Here, $f$ is a given source wavelet. $F_{a}(\vec{k}, \vec{p})$ is an operator, which is acting on $v^{2} \frac{\partial^{2} u}{\partial z^{2}}+v^{2}(1+$ $2 \epsilon) \frac{\partial^{2} u}{\partial x^{2}}$. Since it is a separable approximation of $F$, it can be explicitly represented using Fourier transforms. Let us first denote

$$
m(\vec{x}, \vec{k})=m_{x}(\vec{x}) m_{k}(\vec{k}), \quad n(\vec{x}, \vec{k})=n_{x}(\vec{x}) n_{k}(\vec{k}) .
$$




\begin{tabular}{|c|c|c|c|}
\hline$\Omega$ & $\begin{array}{c}b_{1}, b_{2}, b_{3}, b_{4}, b_{5} \\
b_{6}, b_{7}, b_{8}, b_{9}, b_{10}\end{array}$ & $\begin{array}{c}\text { Maximum error } \\
\text { (optimal coefficients) }\end{array}$ & $\begin{array}{l}\text { Maximum error } \\
\text { (Taylor expansion) }\end{array}$ \\
\hline$-0.2<m<0.2$ & $-0.28661 .04790 .4047 \quad 1.14590 .8203$ & $3.4654 \mathrm{e}-04$ & 0.0977 \\
\hline$n<0.2$ & $\begin{array}{lllll}0.2229 & 0.9568 & -0.2629 & 0.8873 & 0.9126\end{array}$ & & \\
\hline$-0.2<m<0.2$ & -0.30821 .08620 .43211 .29400 .7008 & $8.6873 \mathrm{e}-04$ & 0.0977 \\
\hline$n<0.4$ & 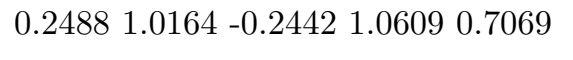 & & \\
\hline$-0.2<m<0.2$ & -0.31941 .10450 .35771 .22930 .6630 & 0.0013 & 0.0977 \\
\hline$n<0.6$ & 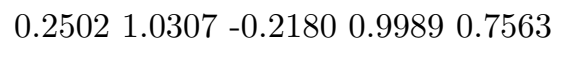 & & \\
\hline$-0.4<m<0.4$ & -0.31201 .10300 .35551 .20890 .7139 & 0.0014 & 0.1916 \\
\hline$n<0.4$ & $\begin{array}{lllll}0.2288 & 0.9825 & -0.2082 & 0.9376 & 0.7461\end{array}$ & & \\
\hline$-0.4<m<0.4$ & -0.32601 .13750 .35301 .32110 .6207 & 0.0024 & 0.1916 \\
\hline$n<0.6$ & 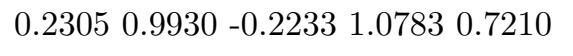 & & \\
\hline$-0.6<m<0.6$ & -0.34391 .19160 .35001 .34910 .6077 & 0.0042 & 0.2825 \\
\hline$n<0.6$ & $\begin{array}{lllll}0.2311 & 1.0283 & -0.2041 & 0.9966 & 0.7358\end{array}$ & & \\
\hline
\end{tabular}

Table 1. A comparison of optimal coefficients and Taylor expansion for different ranges of $\mathrm{m}$ and $\mathrm{n}$.

In the above formulation:

$$
m_{x}(\vec{x})=8(\epsilon-\delta), \quad m_{k}(\vec{k})=\frac{k_{x}^{2} k_{z}^{2}}{\left(k_{x}^{2}+k_{z}^{2}\right)^{2}}, \quad n_{x}(\vec{x})=2 \epsilon, n_{k}(\vec{k})=\frac{k_{x}^{2}}{\left(k_{x}^{2}+k_{z}^{2}\right)} .
$$

According to the definition in (8), and the fact that $m_{k}(\vec{k})$ is always positive, we have

$$
\begin{aligned}
& H(m)\left(b_{1}|m|^{b_{2}}+b_{3}|m|^{b_{4}} n^{b_{5}}\right) \\
= & H\left(m_{x}(\vec{x})\right)\left(b_{1}\left|m_{x}(\vec{x})\right|^{b_{2}} m_{k}(\vec{k})^{b_{2}}+b_{3}\left|m_{x}(\vec{x})\right|^{b_{4}} n_{x}(\vec{x})^{b_{5}} m_{k}(\vec{k})^{b_{4}} n_{k}(\vec{k})^{b_{5}}\right) .
\end{aligned}
$$

Similarly, we have

$$
\begin{aligned}
& (1-H(m))\left(b_{6}|m|^{b_{7}}+b_{8}|m|^{b_{9}} n^{b_{10}}\right) \\
= & \left(1-H\left(m_{x}(\vec{x})\right)\right)\left(b_{6}\left|m_{x}(\vec{x})\right|^{b_{7}} m_{k}(\vec{k})^{b_{7}}+b_{8}\left|m_{x}(\vec{x})\right|^{b_{9}} n_{x}(\vec{x})^{b_{10}} m_{k}(\vec{k})^{b_{9}} n_{k}(\vec{k})^{b_{10}}\right) .
\end{aligned}
$$

Let us denote $f_{1}$ as

$$
f_{1}=v^{2} \frac{\partial^{2} u}{\partial z^{2}}+v^{2}(1+2 \epsilon) \frac{\partial^{2} u}{\partial x^{2}}
$$


Let $\mathbf{F}$ be the Fourier transform operator and $\mathbf{F}^{-1}$ be the inverse Fourier transform operator. Then, $F_{a}(\vec{k}, \vec{p}) f$ can be represented as:

$$
\begin{aligned}
& b_{1} H\left(m_{x}(\vec{x})\right) m_{x}(\vec{x})^{b_{2}} \mathbf{F}^{-1}\left(m_{k}(\vec{k})^{b_{2}} \mathbf{F}\left(f_{1}\right)\right) \\
+ & b_{3} H\left(m_{x}(\vec{x})\right) m_{x}(\vec{x})^{b_{4}} n_{x}(\vec{x})^{b_{5}} \mathbf{F}^{-1}\left(m_{k}(\vec{k})^{b_{4}} n_{k}(\vec{k})^{b_{5}} \mathbf{F}\left(f_{1}\right)\right) \\
+ & b_{6}\left(1-H\left(m_{x}(\vec{x})\right)\right) m_{x}(\vec{x})^{b_{7}} \mathbf{F}^{-1}\left(m_{k}(\vec{k})^{b_{7}} \mathbf{F}\left(f_{1}\right)\right) \\
+ & b_{8}\left(1-H\left(m_{x}(\vec{x})\right)\right) m_{x}(\vec{x})^{b_{9}} n_{x}(\vec{x})^{b_{10}} \mathbf{F}^{-1}\left(m_{k}(\vec{k})^{b_{9}} n_{k}(\vec{k})^{b_{10}} \mathbf{F}\left(f_{1}\right)\right) .
\end{aligned}
$$

Combining Equations (13), (18) and (19), we obtain the approximate continuous form of the acoustic wave equation in VTI media. This continuous equation is no longer a differential equation. However, it is still a linear equation.

\section{NUMERICAL APPROXIMATION OF APPROXIMATE CONTINUOUS EQUATION}

After obtaining the approximate continuous equation, we will discuss the numerical implementation of it. For the time derivative $u_{t t}$, we can use the well-known frog scheme:

$$
u_{t t}\left(t_{n}\right)=\frac{u\left(t_{n+1}\right)-2 u\left(t_{n}\right)+u\left(t_{n-1}\right)}{\Delta t^{2}} .
$$

For the numerical approximation of Equation (18), we can use a regular finite difference approximation of a high order. For the numerical evaluation of $F_{a}(\vec{k}, \vec{p}) f$, we can utilize the Fourier domain since it is a separable approximation. For the case of non-negative or nonpositive $\epsilon-\delta$, we need one forward Fourier transform and two inverse Fourier transforms for each time step according to equation (7). For the case that $\epsilon-\delta$ has both positive and negative values, we need one forward Fourier transform and four inverse Fourier transforms according to equation (8). When $\epsilon=\delta$, no Fourier transforms are required. Based on the above derivation, the resulting number of Fourier transforms is independent on the space dimension and the material parameters. However, we can get a better approximation when $\epsilon-\delta$ and $\epsilon$ are relatively small.

\section{NUMERICAL EXAMPLES}

The first example shows the accuracy of the proposed method for a homogeneous medium. The space sampling is $0.01 \mathrm{~km}$ and the time sampling is $0.001 \mathrm{~s}$. The vertical velocity $v=1.5$ $\mathrm{km} / \mathrm{s}$ and $\epsilon=0.2$. We test different cases in which $\epsilon-\delta$ is positive, negative or zero, and show examples where $\delta=0.4,0.2,0$. The source is a ricker wavelet with the main frequency of 15 


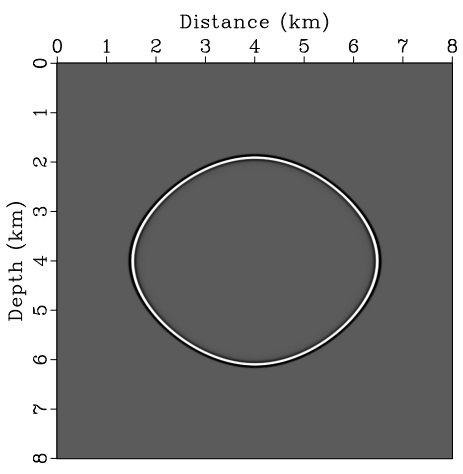

(a)

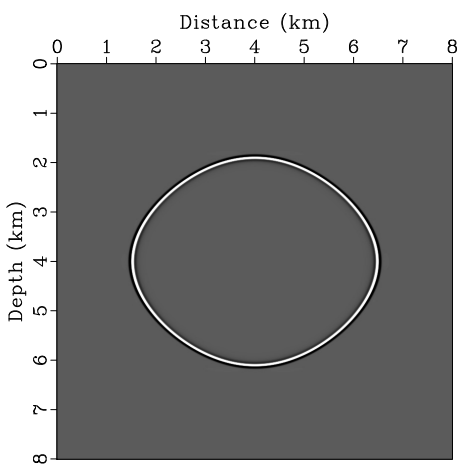

(d)

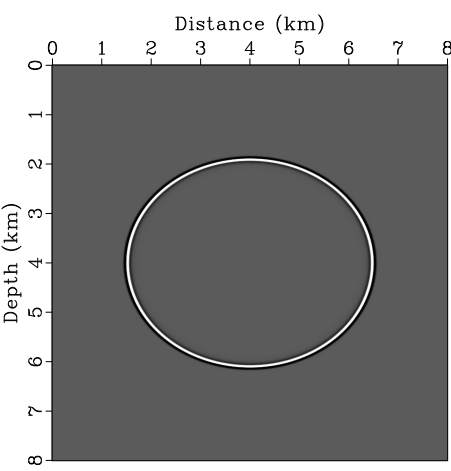

(b)

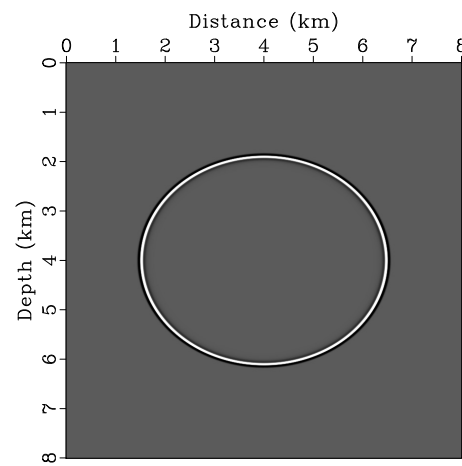

(e)

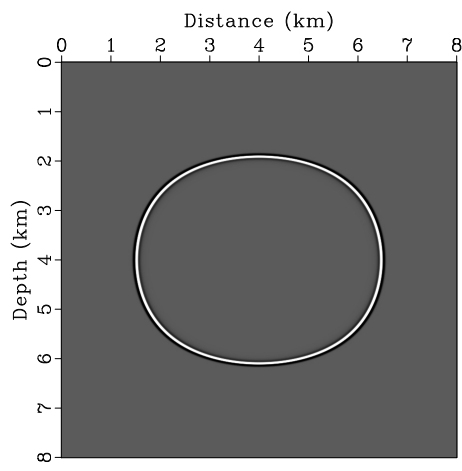

(c)

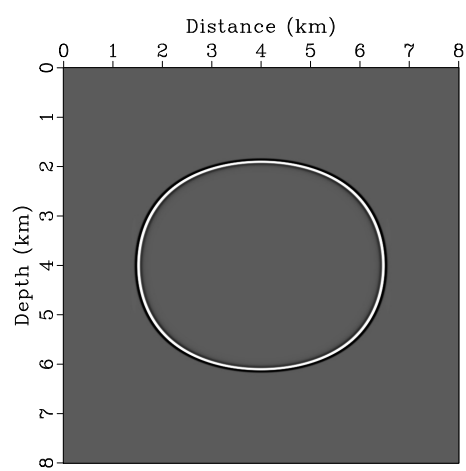

(f)

Figure 2. Synthetic example showing snapshots at $t=1.5 \mathrm{~s}$ for different methods and including (a) the low-rank method for $\delta=0$, (b) the low-rank method for $\delta=0.2$, (c) the low-rank method for $\delta=0.4,(\mathrm{~d})$ the proposed method for $\delta=0$, (e) the proposed method for $\delta=0.2$, and (f) the proposed method for $\delta=0.4$.

$\mathrm{Hz}$ and located in the middle of the model. Figures $2 \mathrm{a}$ and $2 \mathrm{~d}$ show a snapshot at $1.5 \mathrm{~s}$ for the low-rank method and the proposed method for $\delta=0$. In this case, we have $\epsilon-\delta>0$. In the case where $\epsilon-\delta=0$, the snapshots at 1.5 s obtained using the low-rank method and the proposed method are shown in Figures $2 \mathrm{~b}$ and 2e. For $\delta=0.4$ in the case that $\epsilon-\delta<0$, the relative snapshots at $1.5 \mathrm{~s}$ are shown in Figures $2 \mathrm{c}$ and $2 \mathrm{f}$. Results show that our proposed method is reasonably accurate compared to the benchmark result obtained using the lowrank method. To demonstrate the accuracy more clearly, we take one slice of the snapshot for $x=2 \mathrm{~km}$, the results are shown in Figures $3 \mathrm{a}, 3 \mathrm{~b}$ and $3 \mathrm{c}$. With respect to cost, we needed one forward Fourier transform and two inverse Fourier transforms and some extra calculation because $\epsilon-\delta$ is either non-positive or non-negative.

Our next example will show the power of the proposed method in dealing with an interface problem when $\epsilon-\delta>0$ in the top layer and $\epsilon-\delta<0$ in the lower layer. The horizontal velocities $v$ in the top and bottom layers are $1.5 \mathrm{~km} / \mathrm{s}$ and $1.8 \mathrm{~km} / \mathrm{s}$, respectively. The anisotropic 


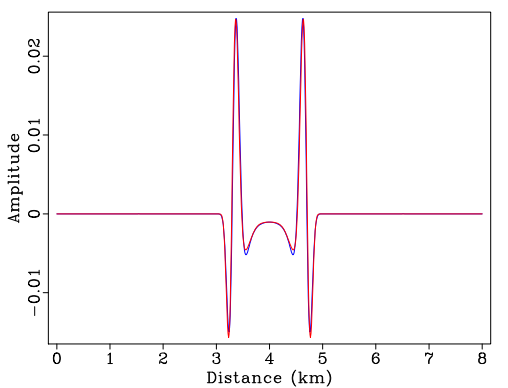

(a)

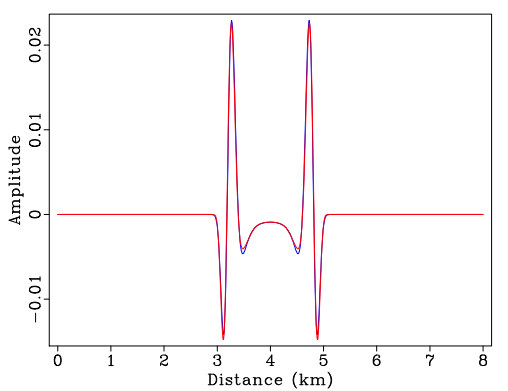

(b)

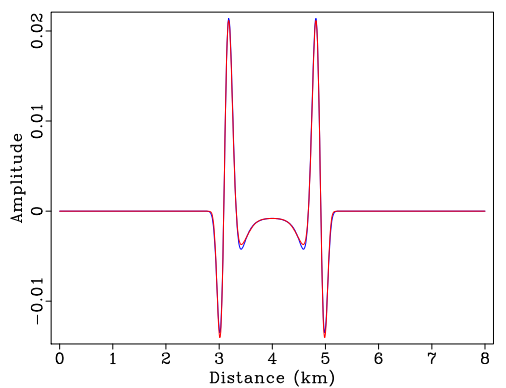

(c)

Figure 3. The slice for $x=2 \mathrm{~km}$ of the snapshot at $t=1.5 \mathrm{~s}$ for different methods and $\delta$ (red curve:low-rank method; blue curve: the proposed method). (a) $\delta=0$; (b) $\delta=0.2$; (c) $\delta=0.4$.

parameters $\epsilon$ in the top and bottom layers are 0.2 and 0.1 , respectively. The anisotropic parameters $\delta$ in the top and bottom layers are 0 and 0.3 , respectively. In this case, the model has $\epsilon-\delta=0.2>0$ in the top layer and $\epsilon-\delta=-0.2<0$ in the bottom layer. We use the space sampling of $0.01 \mathrm{~km}$ and the time sampling is $0.001 \mathrm{~s}$. We place a Ricker wavelet with a peak frequency of $15 \mathrm{~Hz}$ as a source at $x_{s}=4 \mathrm{~km}$ and $z_{s}=3 \mathrm{~km}$. Figure 4a shows a snapshot at time $t=1.5 \mathrm{~s}$ using the low-rank method. Figure $4 \mathrm{~b}$ shows a snapshot at time $t=1.5 \mathrm{~s}$ using the proposed method. To highlight the comparison, the slice at $x=2.5 \mathrm{~km}$ is shown in Figure 5. The cost of our proposed method for this example includes one forward Fourier transform and four inverse Fourier transforms at each time step because $\epsilon-\delta$ has both negative and positive. According to Figure 4b, there are no $S$-wave artifacts even though the source is located in an anisotropic model.

Before investigating a more complicated model, we will show an example of a three dimensional interface problem. We have the same parameters as the previous example where $\epsilon-\delta=0.2>0$ in the upper layer and $\epsilon-\delta=-0.2<0$ in the lower layer. The space sampling is $0.01 \mathrm{~km}$ and the time sampling is $0.001 \mathrm{~s}$. The source is a Ricker wavelet with a peak frequency of $15 \mathrm{~Hz}$ and located in the middle and at a depth of $2 \mathrm{~km}$. Figure 6 shows the snapshot at $t=1 \mathrm{~s}$. It indicates that the proposed method can model the wavefield in three dimensions without $\mathrm{S}$-wave artifacts, even when the source is located in an anisotropic region.

We now consider a more complicated model, which is part of the BP TTI benchmark model. The vertical velocity $v$, and anisotropic parameters $\epsilon$ and $\delta$ are shown in Figures 7a, $7 \mathrm{~b}$ and $7 \mathrm{c}$. To test our algorithm, we modify the model to include $\epsilon-\delta<0$ by adding 0.1 to the original $\delta$ values. We set the water layer be isotropic. We place a source at $z_{s}=4.5 \mathrm{~km}$ and $x_{s}=8 \mathrm{~km}$ using a Ricker wavelet of $10 \mathrm{~Hz}$ peak frequency and use spatial sampling of 0.0125 


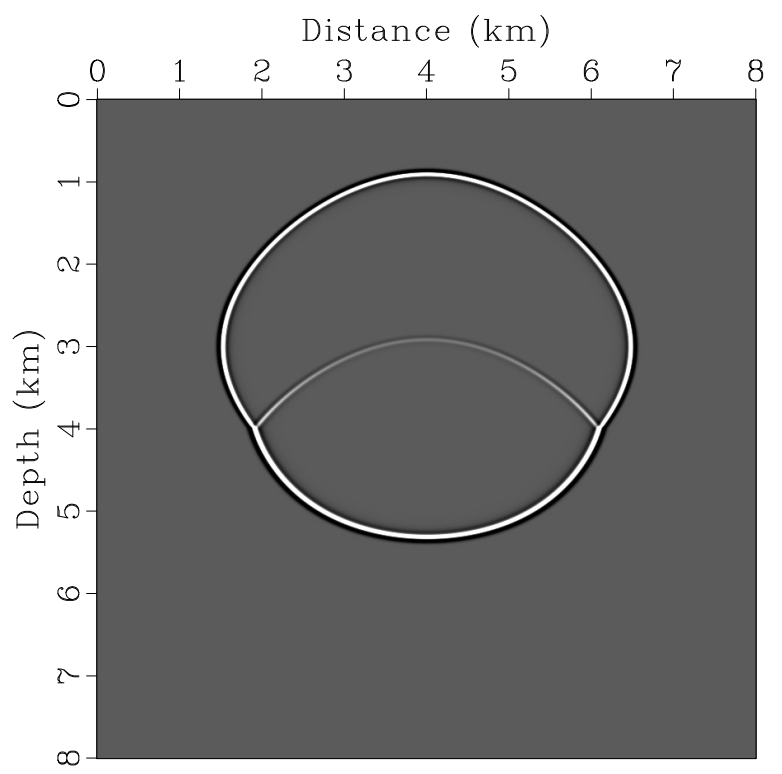

(a)

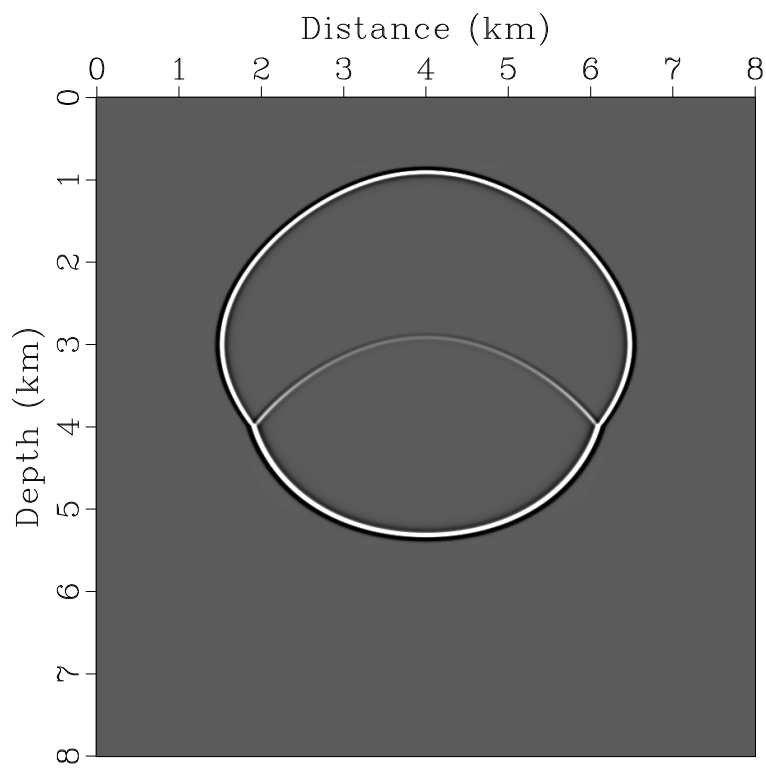

(b)

Figure 4. Wavefield snapshots at $t=1.5 \mathrm{~s}$ for the interface problem for (a) the low-rank method and (b) the proposed method.

$\mathrm{km}$. Wavefield snapshots from the proposed method and the low-rank method in Figures 9 and $8 \mathrm{~b}$ show that the proposed method can also deal with complicated models accurately. In comparison with the low-rank method, we only need one forward Fourier transform and four inverse Fourier transforms instead of one forward Fourier transform and 11 inverse Fourier transforms for the low-rank method.

About the boundary condition, the normal spectral method need to apply a special Fourier transform and special treatment about the absorbing boundary condition(Wu \& Alkhalifah 2016). However, unlike the regular spectral method, this method can deal with free surface boundary condition and absorbing boundary condition easily. Since we are using the standard finite difference method for the operator $f_{1}$ in equation (18), the implementation of free surface boundary condition and absorbing boundary condition similar to standard finite difference method is required only on this operator. This can be explained clearly especially when the boundary is elliptic anisotropic media, because there is no correction for elliptic anisotropic media. To show that the method can deal with the boundary condition, we place the source in the middle of the surface of BP TTI model. And we use the hybrid absorbing boundary condition proposed in Liu \& Sen (2010). And the resulting shot gather is shown in Figure 9.

Finally, we will compare the resulting wavefield of the proposed method for the acoustic wave equation with the wavefield modeled using an elastic wave equation with a more realistic model. Figure 10a shows the $P$-wave velocity. Figures $10 \mathrm{~b}$ and $10 \mathrm{c}$ show the anisotropic 


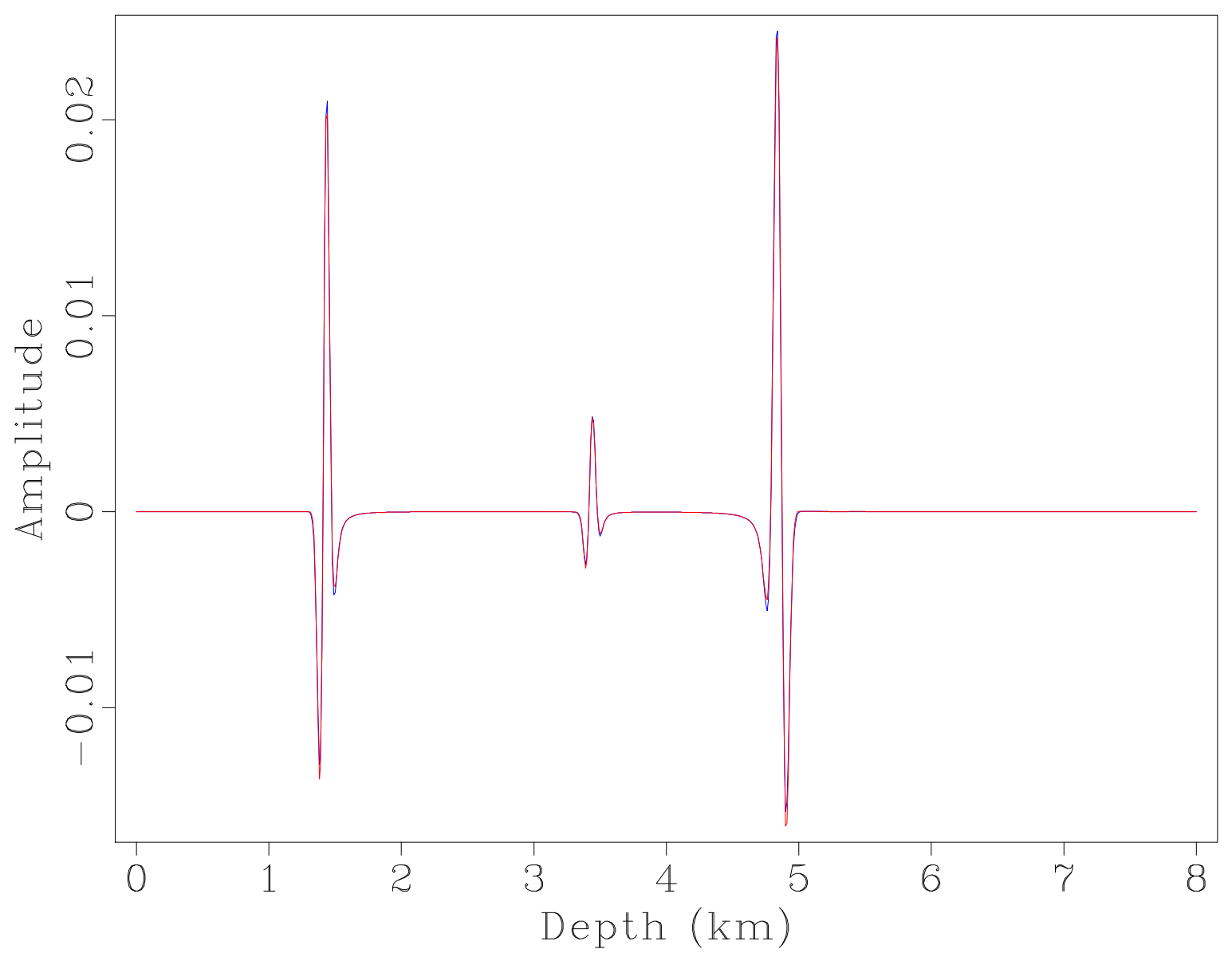

Figure 5. The slice of the the snapshots at $t=1.5 \mathrm{~s}$ for different method (red curve:low-rank method; blue curve: the proposed method).

parameters $\epsilon$ and $\delta$, respectively. The resulting wavefield modeled by the proposed method for the acoustic wave equation is shown in Figure 11a. To evaluate the accuracy of the proposed equation and implementation, we plot the modeled P-wave using elastic modeling shown in Figure 11b. Here, the elastic $P$-wave is obtained by simply taking the divergence of the elastic wavefield. This is a rough approximation in the case of anisotropic media. That is the reason why the resulting wavefield still has $S$-wave artifacts in Figure 11b. Some advanced algorithms (Cheng \& Fomel 2014; Cheng \& Kang 2014; Cheng et al. 2016) can be used to obtain clean $P$-wave from elastic wave result. However, they usually require extra cost. Comparing Figures $11 \mathrm{a}$ and $11 \mathrm{~b}$, we can see that the proposed method is reasonably accurate for a realistic velocity model. 


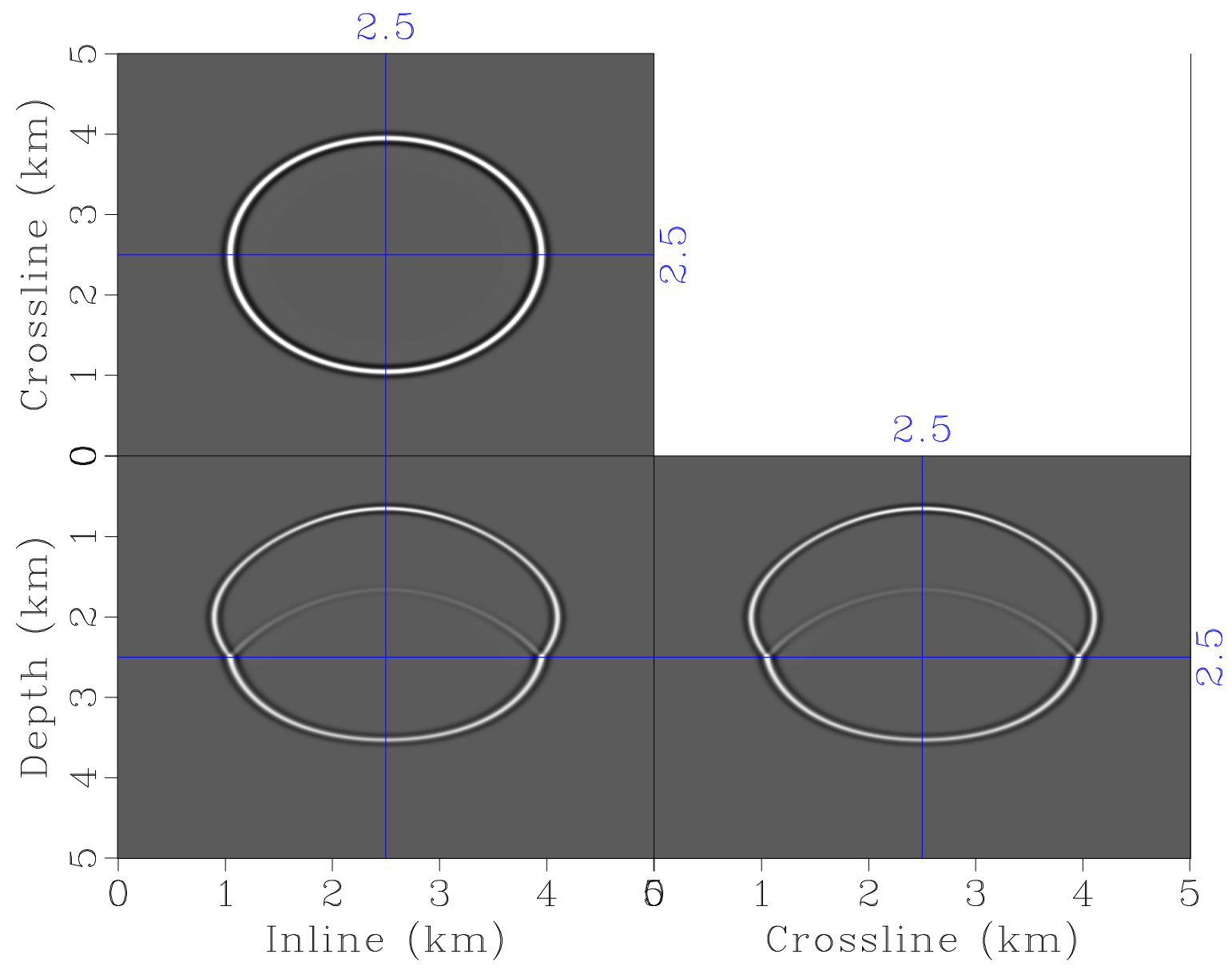

Figure 6. Wavefield snapshots with the proposed method for a three dimensional interface problem.

\section{CONCLUSIONS}

We derived an approximate acoustic wave equation for transversely isotropic media based on a partially separable approximation, especially for the case that $\epsilon-\delta$ can be both positive and negative. This equation admits no $S$-wave artifacts even when the source is located in

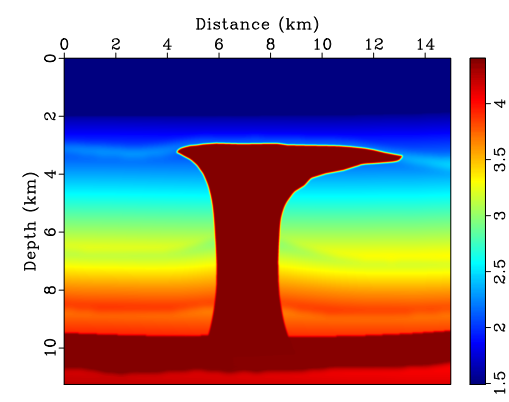

(a)

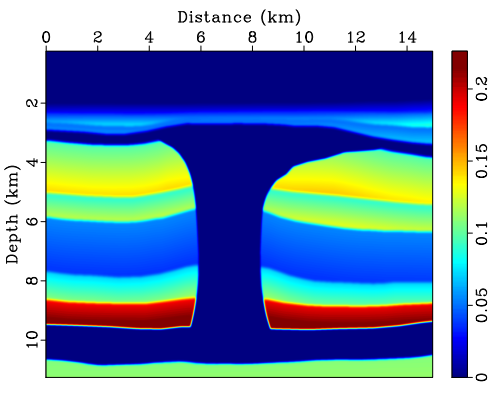

(b)

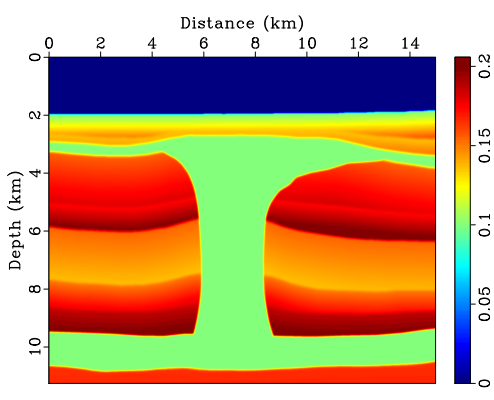

(c)

Figure 7. The BP TTI model parameters including (a) NMO velocity v, (b) $\epsilon$ and (c) $\delta$. 


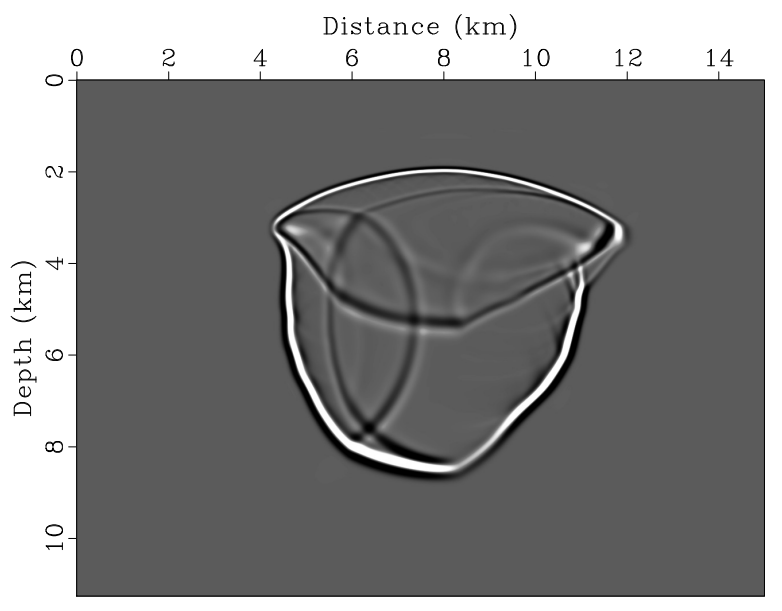

(a)

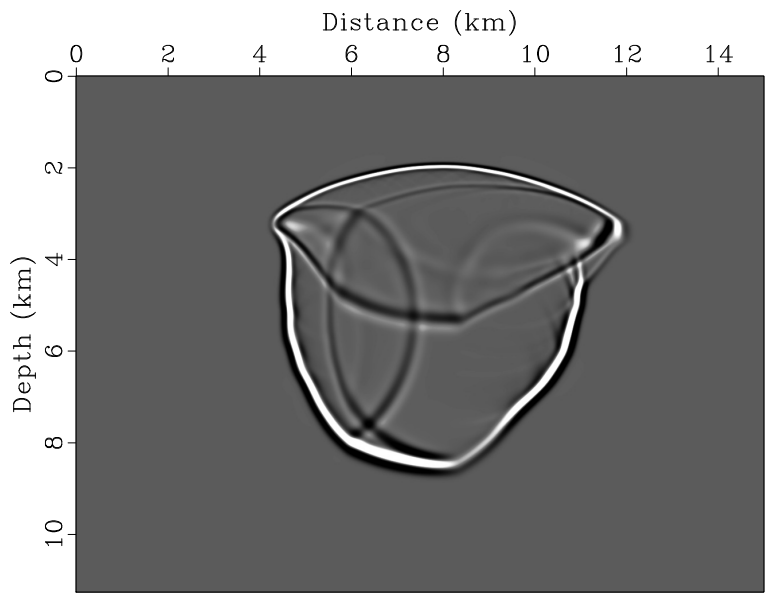

(b)

Figure 8. Wavefield snapshots from the BP TTI model at $1 \mathrm{~s}$ using (a) the proposed method and (b) the low-rank method.

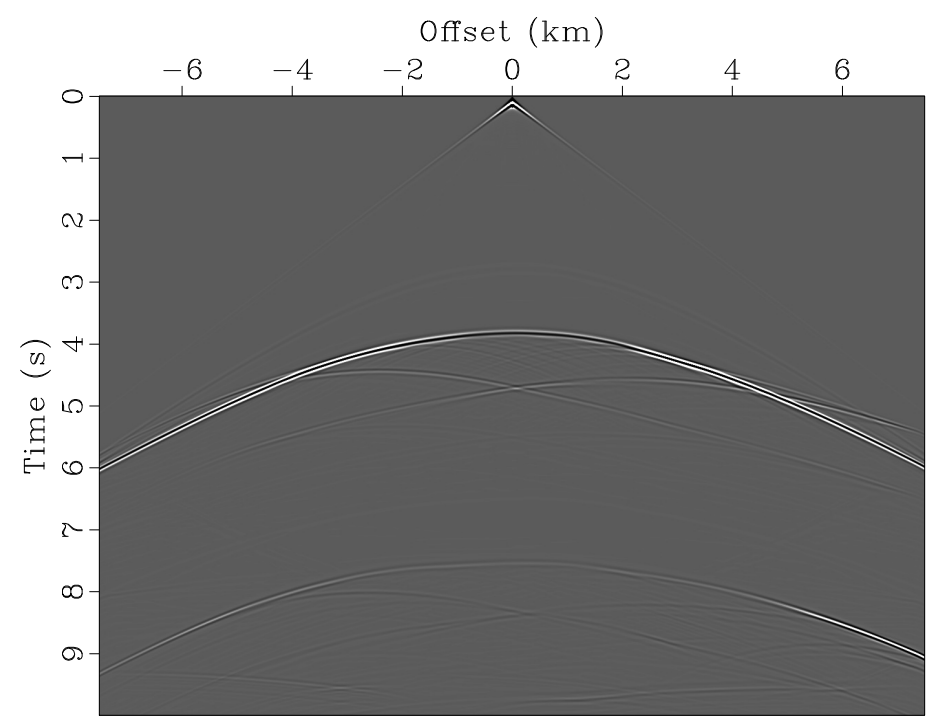

Figure 9. The modelled data on the surface with the source located in the middle of the surface.

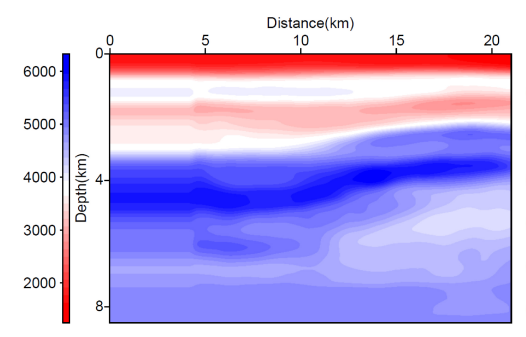

(a)

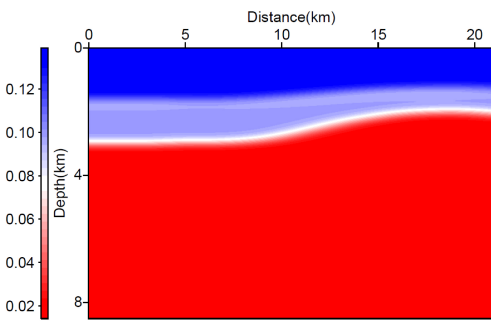

(b)

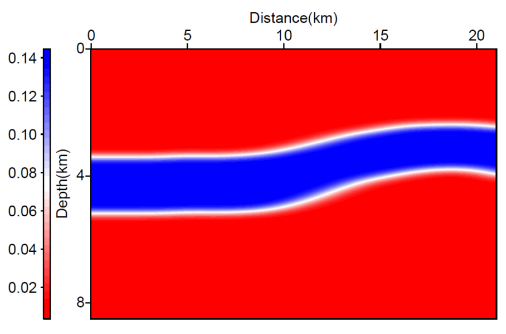

(c)

Figure 10. Parameters for a realistic model including (a) the p-wave velocity, (b) $\epsilon$, and (c) $\delta$. 


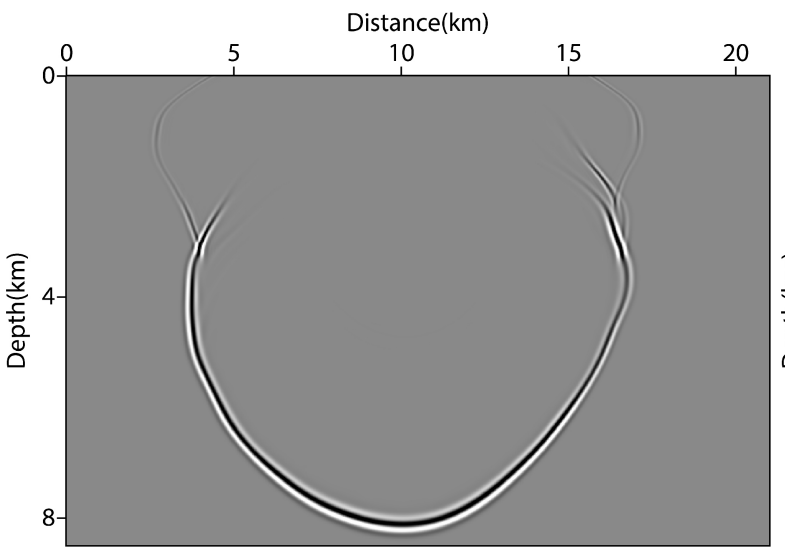

(a)

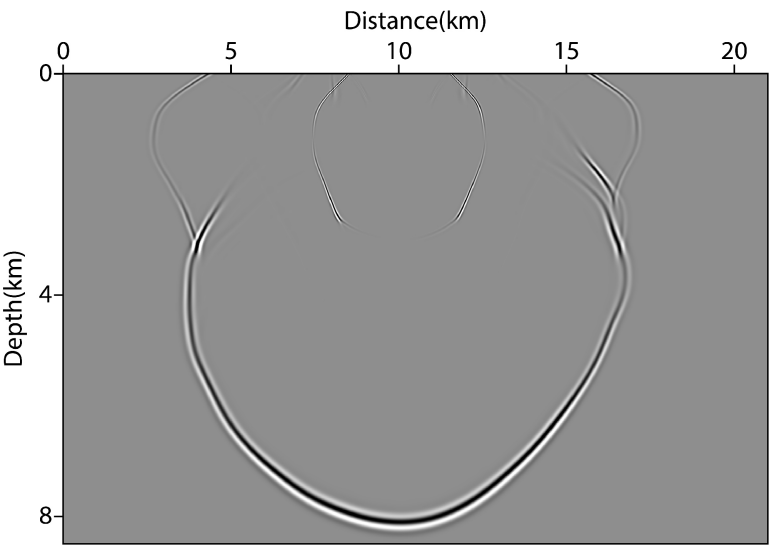

(b)

Figure 11. Wavefield snapshots at $1.7 \mathrm{~s}$ from the realistic model for the proposed method using (a) the acoustic wave equation and (b) elastic modeling.

an anisotropic area. To solve this equation efficiently, we proposed a hybrid implementation approach, which combines the finite difference and spectral methods. For each time step, we require only one forward fast Fourier transform and four inverse Fourier transforms when $\epsilon-\delta$ has both positive and negative values. One forward fast Fourier transform and two inverse Fourier transforms are required when $\epsilon-\delta$ is either only non-positive or only non-negative. Numerical examples show that this method is reasonably accurate and efficient.

\section{ACKNOWLEDGMENTS}

We thank KAUST for its support and the SWAG group for the collaborative environment. We also thank the associate editor Jean Virieux, Hejun Zhu, and another anonymous reviewer for their fruitful suggestions and comments. The research reported in this publication was supported by funding from King Abdullah University of Science and Technology (KAUST). For computer time, this research used the resources of the Supercomputing Laboratory at King Abdullah University of Science and Technology (KAUST) in Thuwal, Saudi Arabia. We also thank Michael Jervis for proofreading the article and BP for providing the BP TTI benchmark model.

\section{REFERENCES}

Alkhalifah, T., 2000. An acoustic wave equation for anisotropic media, Geophysics, 65(4), 1239-1250. Alkhalifah, T., 2014. Effective wavefield extrapolation in anisotropic media: accounting for resolvable anisotropy, Geophysical Prospecting, 62(5), 1089-1099. 
Alkhalifah, T. \& Plessix, R., 2014. A recipe for practical full-waveform inversion in anisotropic media: An analytical parameter resolution study, Geophysics, $\mathbf{7 9 ( 3 ) , ~ R 9 1 - R 1 0 1 . ~}$

Alkhalifah, T. \& Wu, Z., 2016. The natural combination of full and image-based waveform inversion, Geophysical Prospecting, 64(1), 19-30.

Alkhalifah, T., Ma, X., Waheed, U., \& Zuberi, M., 2013. Efficient anisotropic wavefield extrapolation using effective isotropic models, in 75th Annual EAGE Meeting, EAGE, Expanded Abstracts, p. Tu0116.

Baden, D., Henry, P., Saracco, G., Marié, L., \& Guglielmi, Y., 2017. Investigation of anisotropy in nonsaturated and heterogeneous carbonate series using crosshole acoustic techniques, in 87th Annual International Meeting, SEG, Expanded Abstracts, pp. 310-315.

Bakker, P. M. \& Duveneck, E., 2011. Stability analysis for acoustic wave propagation in tilted TI media by finite differences, Geophysical Journal International, 185(2), 911-921.

Bube, K. P., Nemeth, T., Stefani, J. P., Ergas, R., Liu, W., Nihei, K. T., \& Zhang, L., 2012. On the instability in second-order systems for acoustic VTI and TTI media, Geophysics, 77(5), T171-T186.

Cheng, J. \& Fomel, S., 2014. Fast algorithms for elastic-wave-mode separation and vector decomposition using low-rank approximation for anisotropic media, GEOPHYSICS, 79(4), C97-C110.

Cheng, J. \& Kang, W., 2014. Simulating propagation of separated wave modes in general anisotropic media, part i: qp-wave propagators, GEOPHYSICS, 79(1), C1-C18.

Cheng, J., Alkhalifah, T., Wu, Z., Zou, P., \& Wang, C., 2016. Simulating propagation of decoupled elastic waves using low-rank approximate mixed-domain integral operators for anisotropic media, GEOPHYSICS, 81(2), T63-T77.

Cheng, X., Jiao, K., Sun, D., \& Vigh, D., 2014a. Multiparameter full-waveform inversion for acoustic VTI medium with surface seismic data, in 76th Annual EAGE Meeting, EAGE, Expanded Abstracts, p. WeE10604.

Cheng, X., Jiao, K., Sun, D., \& Vigh, D., 2014b. Anisotropic parameter estimation with full-waveform inversion of surface seismic data, in 84th Annual International Meeting, SEG, Expanded Abstracts, pp. $1072-1077$.

da Silva, N. V., Ratcliffe, A., Vinje, V., \& Conroy, G., 2016. A new parameter set for anisotropic multiparameter full-waveform inversion and application to a north sea data set, Geophysics, 81(4), U25-U38.

Du, X., Fletcher, R. P., \& Fowler, P. J., 2010. Pure P-wave propagators versus pseudo-acoustic propagators for RTM in VTI meida, in 72nd Annual EAGE Meeting, EAGE, Expanded Abstracts, p. C013.

Etgen, J. \& Brandsberg-Dahl, S., 2009. The pseudo-analytical method: application of pseudoLaplacians to acoustic and acoustic anisotropic wave propagation, in 79th Annual International Meeting, SEG, Expanded Abstracts, pp. 2552-2556.

Fomel, S., Ying, L., \& Song, X., 2013. Seismic wave extrapolation using lowrank symbol approxima- 
tion, Geophysical Prospecting, 61(3), 526-536.

Gholami, Y., Brossier, R., Operto, S., Ribodetti, A., \& Virieux, J., 2013. Which parameterization is suitable for acoustic vertical transverse isotropic full waveform inversion? part 1: Sensitivity and trade-off analysis, Geophysics, $\mathbf{7 8}(2), \mathrm{R} 81-\mathrm{R} 105$.

Ibanez-Jacome, W., Alkhalifah, T., \& Waheed, U. b., 2014. Effective orthorhombic anisotropic models for wavefield extrapolation, Geophysical Journal International, 198(3), 1653-1661.

Le, H., Biondi, B., Clapp, R. G., \& Levin, S. A., 2015. Using a nonlinear acoustic wave equation for anisotropic inversion, in 85th Annual International Meeting, SEG, Expanded Abstracts, pp. $467-471$

Liu, Y. \& Sen, M. K., 2010. A hybrid scheme for absorbing edge reflections in numerical modeling of wave propagation, GEOPHYSICS, 75(2), A1-A6.

Operto, S., Miniussi, A., Brossier, R., Combe, L., Mtivier, L., Monteiller, V., Ribodetti, A., \& Virieux, J., 2015. Efficient 3-d frequency-domain mono-parameter full-waveform inversion of ocean-bottom cable data: application to valhall in the visco-acoustic vertical transverse isotropic approximation, Geophysical Journal International, 202(2), 1362-1391.

Song, X. \& Alkhalifah, T., 2013. Modeling of pseudoacoustic p-waves in orthorhombic media with a low-rank approximation, Geophysics, 78(4), C33-C40.

Sun, J., Fomel, S., \& Ying, L., 2016. Low-rank one-step wave extrapolation for reverse time migration, Geophysics, 81(1), S39-S54.

Thomsen, L., 1986. Weak elastic anisotropy, GEOPHYSICS, 51(10), 1954-1966.

Waheed, U. b. \& Alkhalifah, T., 2015. An efficient wave extrapolation method for anisotropic media with tilt, Geophysical Prospecting, 63(5), 1126-1141.

Wang, Z., 2002. Seismic anisotropy in sedimentary rocks, part 2: Laboratory data, GEOPHYSICS, 67(5), 1423-1440.

Wu, Z. \& Alkhalifah, T., 2014. The optimized expansion based low-rank method for wavefield extrapolation, Geophysics, 79(2), T51-T60.

Wu, Z. \& Alkhalifah, T., 2015. Simultaneous inversion of the background velocity and the perturbation in full-waveform inversion, GEOPHYSICS, 80(6), R317-R329.

Wu, Z. \& Alkhalifah, T., 2016. The optimized gradient method for full waveform inversion and its spectral implementation, Geophysical Journal International, 205(3), 1823-1831.

Wu, Z. \& Alkhalifah, T., 2017. Efficient scattering-angle enrichment for a nonlinear inversion of the background and perturbations components of a velocity model, Geophysical Journal International, 210(3), 1981-1992.

Wu, Z. \& Alkhalifah, T., 2018. An efficient helmholtz solver for acoustic transversely isotropic media, GEOPHYSICS, 83(2), C75-C83.

Xu, S. \& Zhou, H., 2014. Accurate simulations of pure quasi-p-waves in complex anisotropic media, Geophysics, 79(6), T341-T348. 
Xu, S., Tang, B., Mu, J., \& Zhou, H., 2015. Quasi-p wave propagation with an elliptic differential operator, in 85th Annual International Meeting, SEG, Expanded Abstracts, pp. 4380-4384.

Zhang, Y., Zhang, H., \& Zhang, G., 2011. A stable TTI reverse time migration and its implementation, Geophysics, $\mathbf{7 6}(3)$, WA3-WA11.

Zhang, Z.-D., Liu, Y., Alkhalifah, T., \& Wu, Z., 2017. Efficient anisotropic quasi-p wavefield extrapolation using an isotropic low-rank approximation, Geophysical Journal International, p. ggx543.

Zhou, H., Zhang, G., \& Bloor, R., 2006. An anisotropic acoustic wave equation for VTI media, in 68th Annual EAGE Meeting, EAGE, Expanded Abstracts, p. H033. 